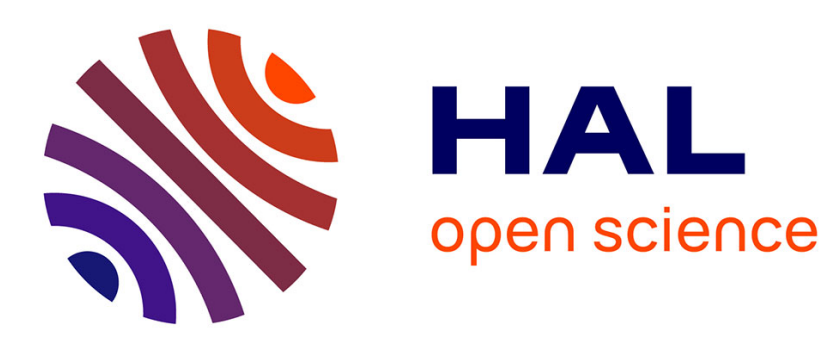

\title{
Rotational echoes as a tool for investigating ultrafast collisional dynamics of molecules
}

\author{
H. Zhang, B. Lavorel, F. Billard, J.-M. Hartmann, E. Hertz, O. Faucher, \\ Junyang Ma, Jian Wu, Erez Gershnabel, Yehiam Prior, et al.
}

\section{To cite this version:}

H. Zhang, B. Lavorel, F. Billard, J.-M. Hartmann, E. Hertz, et al.. Rotational echoes as a tool for investigating ultrafast collisional dynamics of molecules. Physical Review Letters, 2019, 122 (19), 10.1103/PhysRevLett.122.193401 . hal-02398543

\section{HAL Id: hal-02398543 \\ https://hal.science/hal-02398543}

Submitted on 7 Dec 2019

HAL is a multi-disciplinary open access archive for the deposit and dissemination of scientific research documents, whether they are published or not. The documents may come from teaching and research institutions in France or abroad, or from public or private research centers.
L'archive ouverte pluridisciplinaire HAL, est destinée au dépôt et à la diffusion de documents scientifiques de niveau recherche, publiés ou non, émanant des établissements d'enseignement et de recherche français ou étrangers, des laboratoires publics ou privés. 


\title{
Rotational echoes as a tool for investigating ultrafast collisional dynamics of molecules
}

\author{
H. Zhang ${ }^{1}$, B. Lavorel ${ }^{1}$, F. Billard ${ }^{1}$, J.-M. Hartmann ${ }^{2}$, E. Hertz ${ }^{1}$, and O. Faucher ${ }^{1 *}$ \\ ${ }^{1}$ Laboratoire Interdisciplinaire CARNOT de Bourgogne, \\ UMR 6303 CNRS-Université de Bourgogne, BP 47870, 21078 Dijon, France and \\ ${ }^{2}$ Laboratoire de Météorologie Dynamique/IPSL, CNRS, \\ École polytechnique, Sorbonne Université, École Normale Supérieure, \\ PSL Research University, F-91120 Palaiseau, France
}

\author{
Junyang $\mathrm{Ma}^{1,3}$ and Jian $\mathrm{Wu}^{3 \dagger}$ \\ ${ }^{3}$ State Key Laboratory of Precision Spectroscopy, \\ East China Normal University, Shanghai 200062, China
}

\author{
Erez Gershnabel ${ }^{4}$, Yehiam Prior ${ }^{3,4}$, and Ilya Sh. Averbukh ${ }^{4 \ddagger}$ \\ ${ }^{4}$ AMOS and Department of Chemical and Biological Physics, \\ The Weizmann Institute of Science, Rehovot 76100, Israel
}

(Dated: December 7, 2019)

\begin{abstract}
We show that recently discovered rotational echoes of molecules provide an efficient tool for studying collisional molecular dynamics in high-pressure gases. Our study demonstrates that rotational echoes enable the observation of extremely fast collisional dissipation, at time scales of the order of few picoseconds, and possibly shorter. The decay of the rotational alignment echoes in $\mathrm{CO}_{2}$ gas and $\mathrm{CO}_{2}$-He mixture up to 50 bar was studied experimentally, delivering collision rates that are in good agreement with the theoretical expectations. The suggested measurement protocol may be used in other high-density media, and potentially in liquids.
\end{abstract}

PACS numbers: 45.50.-j, 37.10.Vz, 42.50.Md

Echo phenomenon is well known in various domains of physics, ranging from NMR spectroscopy [1,2] and nonlinear optics $[3,4]$ to plasma physics $[5,6]$ and physics of particle accelerators [7, 8]. The effect relates to the impulsive stimulation of an ensemble of slightly non-identical nonlinear objects (interacting or non-interacting). A single stimulus induces a coherent collective response signal that dies quickly due to the dispersion in the properties of individual objects (inhomogeneous broadening). The second stimulus applied with a delay $\tau_{12}$ results in a similar fast disappearing coherent response. However, if one continues observing the system for an additional time period of $\tau_{12}$, the system produces another impulsive collective signal (echo) without any further stimulation. Moreover, in some cases, further echoes may be observed after waiting for more time $\left(2 \tau_{12}, 3 \tau_{12}\right.$, etc.). The echo is essentially a classical phenomenon that should be distinguished from the quantum revivals [9-12]. In addition to the fundamental importance of this generic effect, the echoes found multiple applications, mostly as a platform for measuring dephasing processes caused by irreversible dissipation. Recently, a new kind of echoes was discovered in the rotational motion of molecules, the so-called rotational alignment echoes, including regular, high-order and fractional echoes [13, 14], rotated and imaginary echoes [15, 16], and the echo-enabled rephasing of centrifugal distortions [17].

The present Letter describes the first study related to ultrafast collisional dissipation of the rotational echoes generated in high-pressure molecular gases. Decay of the rotational revivals have been successfully used in the past to investigate the collisional dynamics in low pressure gases $[18,19]$, however this approach becomes completely inefficient when the average time between collisions is comparable, or shorter than the rotational revival time. As shown in this letter, the rotational alignment echoes provide a powerful tool for studying collisional effects in dense gases, and, potentially, in liquids.

Traditional echo spectroscopies like spin-echo [1, 2] and photon-echo $[3,4]$ disengage the homogeneous broadening from the overwhelming inhomogeneous broadening of investigated transitions by recording the corresponding echo response as a function of the delay between two excitation pulses. In these cases, the amplitude of the echoes is practically independent of the delay between the kicking stimuli (pulses) when the homogeneous broadening is negligible. Therefore, any decay of the echo amplitude directly reflects the irreversible dephasing.

The mechanism of the echoes in molecular alignment is quite different from the spin- or photon-echo, which makes them sensitive to both the pulse intensities, and to the delay $\tau_{12}$ [13]. Previous investigations on the rotational alignment echoes used rarified gases, and were focused on measuring the temporal and spatial evolution of echoes induced by two laser pulses $\left(\mathcal{P}_{1}\right.$ and $\left.\mathcal{P}_{2}\right)$ separated by a delay $\tau_{12}$. The experimental results clearly demonstrated strong dependence of the echo's amplitude on the intensity of the second pulse, and on the delay $\tau_{12}$. As shown in [13], for each $\tau_{12}$ there exists an optimal in- 
tensity of $\mathcal{P}_{2}$ that produces the strongest echo. A recent study [20] arrived at similar conclusions by studying the corresponding echo response in low pressure OCS gas. This behavior is well explained by the classical analysis of rotational echoes based on the kick-induced filamentation of phase space of 2D rigid-rotors [13, 14]. Moreover, such a behavior is also typical for a related problem of beam echoes in particle accelerators [7, 8], in which the phenomenon of phase space filamentation plays an important role too.

In high-pressure gases, alignment echo spectroscopy requires developing a special measurement protocol for disentangling the collision-free dynamics of the echo amplitude from the effects of irreversible dissipation and dephasing in the molecular rotation dynamics. In order to define such a measurement strategy, we first conducted a series of simulations at collision-free conditions, by solving the Liouville-von Neumann equation for $\mathrm{CO}_{2}$ molecules kicked by two non-resonant pulses through the anisotropic polarizability interaction. A temporal trace for the alignment metric $\left(\left\langle\cos ^{2}(\theta)\right\rangle-1 / 3\right)[21]$ of $\mathrm{CO}_{2}$ molecules $(\theta$ is the angle between the molecular axis and the direction of polarization of $\mathcal{P}_{1}$ and $\mathcal{P}_{2}$ ) is shown in Fig. 1 (a), where the alignment responses induced around $\mathcal{P}_{1}$ and $\mathcal{P}_{2}$, the rotational echo (E) at $2 \tau_{12}$, the secondorder rotational echo $(\mathrm{sE})$ at $3 \tau_{12}$, and the quarter-revival of the imaginary echo (iE) at $T_{\text {rev }} / 4-\tau_{12}\left(T_{\text {rev }}=42.7 \mathrm{ps}\right.$ is the revival time of $\mathrm{CO}_{2}$ molecules), are depicted with different colors. The strength of the rotational echo is characterized by the peak-to-dip amplitude as $\mathcal{S}_{\text {echo }}$. It is clearly inferred from Figs. 1(c)-(d) that both the optimal intensity $I_{2}$ of $\mathcal{P}_{2}$ and optimal delay $\tau_{12}$ are well defined: the optimal $I_{2}$ can be found for each $\tau_{12}$ (consider a vertical straight-cut of each contour-plot) and similarly the optimal $\tau_{12}$ can be found for each $I_{2}$ (consider a horizontal straight-cut of each contour-plot). The black dotdashed lines connect the optimal values of $I_{2}$ for varying $\tau_{12}$, featuring that shorter delays necessitate higher $\mathcal{P}_{2}$ intensities to get the echo best-rephased, and, alternatively, larger $I_{2}$ require shorter delays to maximize the echo. Therefore, one cannot simply find a unique optimal intensity for all the delays in rotational echoes, in sharp contrast, say to photon echoes where the optimal strength of the second pulse always corresponds to a $\pi$ pulse [4]. It is worth to mention that the temporal profile of the rotational echo can be even reversed at higher than optimal intensities (represented by negative values plotted in Figs. 1 (c)-(d)), and the 2nd optimal $I_{2}$ for the reversed echoes can be similarly defined (red dashed lines). Moreover, the dependence of rotational echoes on the intensity $I_{1}$ of $\mathcal{P}_{1}$ can be globally factorized out given the same shape of the contour-plots obtained at two distinct values of $I_{1}$ shown in Figs. 1 (c)-(d) with only the increment of color-scales. This entangled dependence of rotational echoes on $I_{2}$ and $\tau_{12}$ is also well captured by the classical model of 2D rigid rotors [13], where the echo
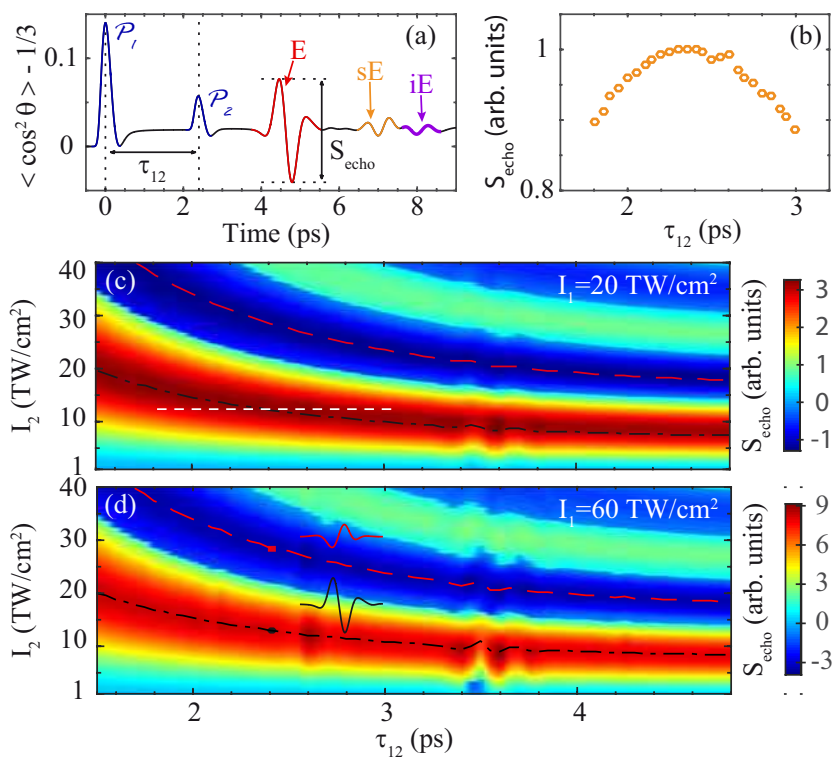

FIG. 1: (color online) (a) Temporal trace of the alignment signal of $\mathrm{CO}_{2}$ kicked by two pump pulses $\left(\mathcal{P}_{1}\right.$ and $\left.\mathcal{P}_{2}\right)$ in the collision-free conditions depicting the echo (E), the secondorder echo (sE), and the imaginary echo (iE). (b) Variation of the echo amplitude along the horizontal dashed-line depicted in (c). Calculated peak-to-dip amplitude of the rotational echo of $\mathrm{CO}_{2}$, as a function of the delay $\tau_{12}$ and intensity of $\mathcal{P}_{2}$, as shown for two intensities of $\mathcal{P}_{1}: I_{1}=20 \mathrm{TW} / \mathrm{cm}^{2}$ (c) and $\mathrm{I}_{1}=60 \mathrm{TW} / \mathrm{cm}^{2}(\mathrm{~d})$.

response is determined by the product of $I_{2} \cdot \tau_{12}=\alpha$, where $\alpha$ is a constant independent of $I_{1}$. Finally, note that the maximal echo amplitudes, obtained by adjusting $I_{2}$ at each delay, are identical with less than $4 \%$ variations for the same $I_{1}$ inside the investigated range of $\tau_{12}$ (1.5-4.8 ps), except for a local "perturbation" occurring around $\tau_{12} \approx 3.6$ ps in Figs. 1 (c)-(d). The latter can be attributed to the temporal coincidence, for this delay, between the full rotational echo (which appears at $t=2 \tau_{12}$ after $P_{1}$ ) and the quarter revival of the imaginary echo (which appears at $T_{\text {rev }} / 4-\tau_{12}=10.7 \mathrm{ps}-\tau_{12}$ ), as explained in more details in the Supplemental Material [22].

Based on the numerical results discussed above, two strategies can be envisaged to utilize the rotational echoes to measure the ultrafast collisional dissipation of molecular rotors at high densities (high pressures), where quantum revivals of molecular alignment induced by a single pulse are hardly exploitable because of their long revival times [19]. The first one is to measure the maximum amplitudes of echoes generated at each delay $\tau_{12}$ by adaptive optimization of the corresponding intensity $I_{2}[20]$. However, a systematic adjustment of intensity at different delays can introduce some experimental uncertainties, especially in high-density samples where the quickly-damped amplitude of the echo signal tends to 


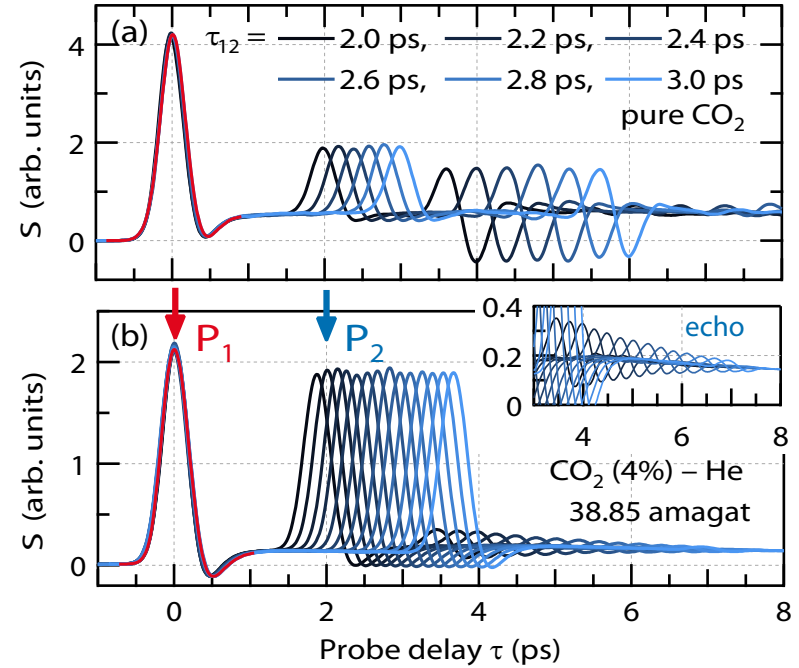

FIG. 2: (color online): (a) Low-pressure temporal traces of the echo recorded in $\mathrm{CO}_{2}$ for different time delays $\tau_{12}$ between the pulse $\mathcal{P}_{1}$ and $\mathcal{P}_{2}$ as a function of the probe delay $\tau$ defined with respect to $\mathcal{P}_{1}$. The intensity of $\mathcal{P}_{2}$ is set to the optimal value for the delay $\tau_{12}=2.4 \mathrm{ps}$, i.e., $13 \mathrm{TW} / \mathrm{cm}^{2}$ (see text). (b) High-pressure temporal traces in $\mathrm{CO}_{2}-\mathrm{He}$ gas mixture at a pressure of 43.5 bar with a zoom-in of the echo decay shown in the inset. The estimated intensity of $\mathcal{P}_{1}$ is $20 \mathrm{TW} / \mathrm{cm}^{2}$. The positions of the alignment peaks respective to $\mathcal{P}_{1}$ and $\mathcal{P}_{2}$ are marked with arrows.

degrade the judicious control of the intensity. The other strategy is to find a compromised and fixed intensity $I_{2}$ for which the echo amplitude varies little over a limited range of $\tau_{12}$ retained for experiments. This is the strategy effectively employed in this work to interrogate the collisional decay of rotational echoes of $\mathrm{CO}_{2}$ molecules, at high densities and/or diluted with high pressure Helium. The compromised intensity $I_{2}$ is found around $13 \mathrm{TW} / \mathrm{cm}^{2}$, which renders the amplitudes of rotational echoes relatively uniform with less than $10 \%$ variations, for delays $\tau_{12}$ scanned between 1.8 and 3.0 ps in collisionfree conditions, as shown in Fig. 1(b).

The rotational echoes are measured by using a balanced detection of time-resolved birefringence of gas phase molecules impulsively aligned by two successive laser kicks. Details about the experimental setup are provided in the Supplemental Material [22]. Briefly, two collinear amplified 100 fs laser pulses with parallel linear polarizations, temporally separated by the adjustable delay $\tau_{12}$, are tightly focused in a high-pressure static cell specially designed for polarization resolved highpressure measurements. The transient birefringence resulting from the ultrafast anisotropic redistribution of molecule orientations is decoded by a probe pulse polarized at $45^{\circ}$ with respect to $\mathcal{P}_{1}$ and $\mathcal{P}_{2}$. The probe beam is spatially overlapped with the two collinear pump beams in the focus, with a small crossing angle. The modifi- cation of the polarization of the probe pulse is analysed with a highly sensitive balanced detector providing a heterodyne signal

$$
\mathcal{S}\left(\tau_{12}, \tau\right) \propto \int_{-\infty}^{\infty} I_{3}(t-\tau)\left[\left\langle\cos ^{2}(\theta)\right\rangle\left(t, \tau_{12}\right)-\frac{1}{3}\right] d t,
$$

where $\left\langle\cos ^{2}(\theta)\right\rangle\left(t, \tau_{12}\right)$ is the alignment metric as shown in Fig. $1(\mathrm{a})$ and $I_{3}$ is the probe intensity. The ultrafast dissipation of molecular alignment is inferred from the decay of the rotational echo observed by scanning the delay $\tau$ between the probe pulse and $\mathcal{P}_{1}$ for different fixed values of the delay $\tau_{12}$ between $\mathcal{P}_{1}$ and $\mathcal{P}_{2}$. Two molecular systems were investigated: pure $\mathrm{CO}_{2}$ and $\mathrm{CO}_{2}-\mathrm{He}$ mixture. These systems are of interest because of their well documented collisional decay rates from both experiment and theory (see, e.g., [18, 19] and those cited in the discussion of line broadening data at the end of this Letter). Additionally, reliable intermolecular potentials, essential ingredients of the theoretical simulations (see below), are available for the involved collisional pairs. Regarding pure $\mathrm{CO}_{2}$, it should be noted that the high peak powers required for the observation of the rotational echoes lead to nonlinear propagation effects for high gas densities, which are not observed for $\mathrm{CO}_{2}$ diluted in $\mathrm{He}$ in the same density range, due to the much weaker nonlinear polarizability of He compared to $\mathrm{CO}_{2}$ [23]. Considering the investigated pressure range and the tight-focusing geometry of the experiment, we observed that the propagation effects affect the intensity of $\mathcal{P}_{2}$ by $\approx 30 \%$ in the overlapping region of the three pulses. Since the amplitude of the echo is strongly dependent on the $\mathcal{P}_{2}$ intensity, the energy of $\mathcal{P}_{2}$ delivered by the laser was corrected by optimizing the peak-to-dip amplitude of the echo signal at $\tau_{12}=2.4$ ps for each gas pressure so as to keep the intensity in the probe gas volume close to the compromised value. In contrast, the energy of $\mathcal{P}_{1}$ was not corrected as its intensity does not affect the $\tau_{12}$ dependence of the echo amplitude, as shown in Figs. 1 (c)-(d). Finally, note that the plasma contribution to the relaxation of the echo can be neglected, considering the very low relative density of the plasma, guaranteed by the low intensities of $\mathcal{P}_{1}$ and $\mathcal{P}_{2}$ used in the experiment, as well as the small scattering cross-sections for electron collisions with $\mathrm{CO}_{2}$ [24].

In parallel with the experiments, theoretical calculations were also performed using Classical Molecular Dynamics Simulations (CMDS). These simulations, carried following Ref. [18], provide the time dependence of $\left\langle\cos ^{2}(\theta)\right\rangle(t)$ for given values of $I_{1}, I_{2}, \tau_{12}$, and gas density. They were performed, free of any adjustable parameters, as detailed in the Supplemental Material [22].

In a first step, the strategy introduced in Fig. 1 was tested experimentally. Typical results are presented in Fig. 2 (a) where a series of temporal traces of molecular alignment is recorded for different delays $\tau_{12}$ and for an intensity $I_{2}$ set around $13 \mathrm{TW} / \mathrm{cm}^{2}$. This value was 
found by optimizing the amplitude of the echo at $\tau_{12}=2.4$ ps. These measurements were conducted at room temperature in pure $\mathrm{CO}_{2}$ kept at low pressure in order to avoid any noticeable collisional decay of the echo signal within the investigated temporal range $\Delta \tau$. In consequence, the slight change of the echo amplitude observed with respect to $\tau_{12}$ is mainly due to the inherent response of single molecules and also to the stability of the experiment. In agreement with the theoretical predictions of Fig. 1 (b), the variation of the echo-amplitudes over the investigated range of $\tau_{12}$ is less than $10 \%$.
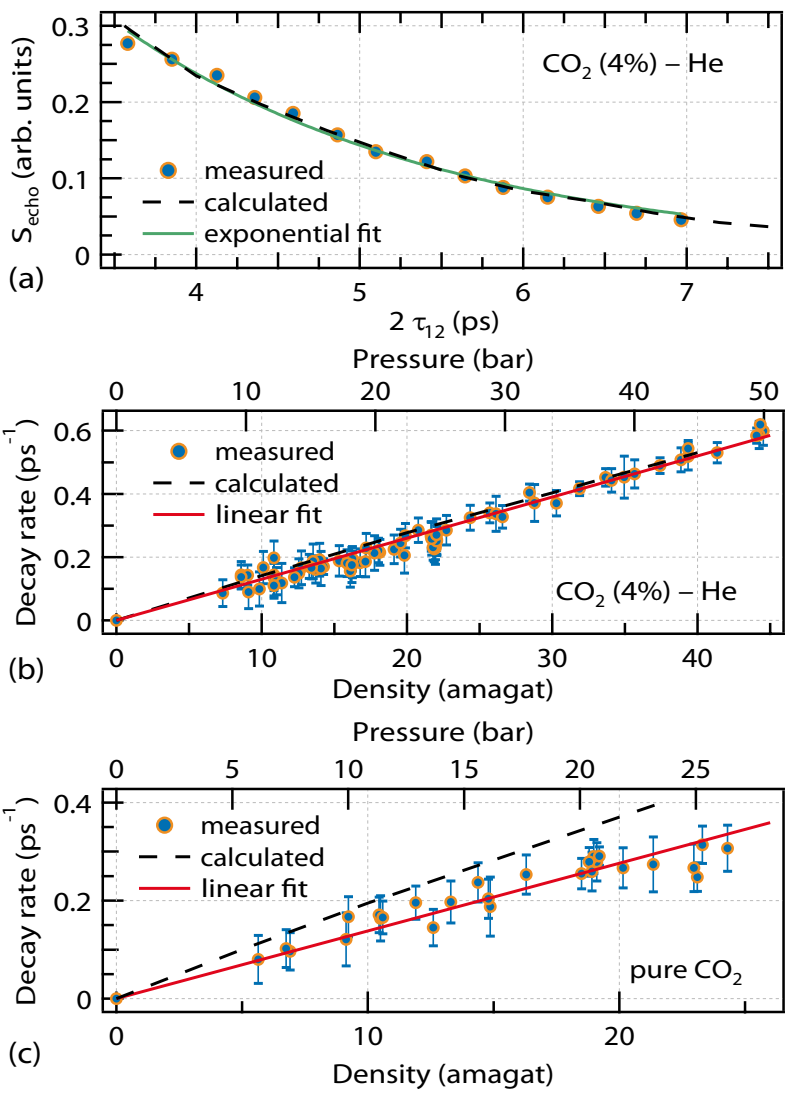

FIG. 3: (color online) (a) Peak-to-dip amplitude of the echo (filled circles) recorded in a $\mathrm{CO}_{2}-\mathrm{He}$ gas mixture $(\mathrm{P}=43.5 \mathrm{bar})$ as a function of $\tau_{12}$ compared with the CMDS simulations (dashed line). Least-squares fitting of the experimental data with an exponential law (solid line). (b) Decay rates $\gamma$ of the echo (filled circles) measured for various density of the gas mixture with the linear fit (solid line) of the data and the CMDS calculations (dashed line). The error bars reflect the dispersion of the measurements. The bottom (top) $\mathrm{x}$-axis denotes the gas density (pressure) in units of amagat (bar). (c) Decay rates $\gamma$ measured in pure $\mathrm{CO}_{2}$.

Next, echoes of $\mathrm{CO}_{2}$ were recorded at the pressure of 43.5 bar in a $\mathrm{CO}_{2}$-He mixture over a temporal probe window $\Delta \tau=9 \mathrm{ps}$, using the same strategy as in Fig. 2 (a). Note that for such high pressure, the alignment revivals of $\mathrm{CO}_{2}$ separated by $T_{\mathrm{rev}} / 4 \approx 10.7$ ps cannot be properly
TABLE I: Measured and calculated collisional decay time constants $\tau_{\mathrm{E}}$ and $\tau_{\mathrm{R}}$ in ps.amagat units for infinitely diluted $\mathrm{CO}_{2}-$ $\mathrm{He}$ gas mixture and pure $\mathrm{CO}_{2}$ gas. The numbers in parenthesis correspond to three standard deviations.

\begin{tabular}{ccccc}
\hline \hline & \multicolumn{2}{c}{$\mathrm{CO}_{2}-\mathrm{He}$} & \multicolumn{2}{c}{$\mathrm{CO}_{2}$} \\
& Echo & Revivals & Echo & Revivals \\
\hline Experiment & $77(4)$ & $83(8)[19]$ & $73(7)$ & $68(7)[19]$ \\
\hline CMDS Model & $74(7)$ & $81[18]$ & $53(2)$ & $57[18]$ \\
\hline \hline
\end{tabular}

used anymore for tracking collisional processes [19, 25]. As shown in Fig. 2 (b), the collisional dissipation of the echo is evidenced by varying the delay $\tau_{12}$. The corresponding peak-to-dip echo-amplitudes are plotted in Fig. 3(a). We apply to the data an exponential leastsquares fit $\exp \left(-2 \tau_{12} \gamma\right)$, where $\gamma$ defines the relaxation rate of the echo estimated at a given density. As can be seen, the agreement between CMDS-predicted and measured results is excellent in this particular case (see below for a further discussion).

In order to assess the consistency of our measurements, we report in Fig. 3(b) the collisional decay rate $\gamma$ of the echo determined for different gas densities of the mixture (expressed hereafter in amagat units) corresponding to pressures ranging from 8 to 50 bar. Remind that in the binary collision regime, which is valid in this pressure range, the collisional relaxation rate of the alignment signal should be proportional to the number density. We can see from the linear fit of the data that this is well verified for the measured echoes as also evidenced by the results of the CMDS model presented on the same figure. The experimental value of the decay time constant of the echo $\tau_{\mathrm{E}}=\gamma_{0}^{-1}$, with $\gamma_{0}$ the slope versus density extracted from the linear least-squares fitting of the data, is reported in Table I together with the value predicted by the CMDS model. As shown, the experimental observation ( $\tau_{\mathrm{E}}=77$ ps.amagat) is well reproduced by the theory $\left(\tau_{\mathrm{E}}=74\right.$ ps.amagat). For comparison, we also provide in the same table the decay time constant of the alignment revivals of $\mathrm{CO}_{2}\left(\tau_{\mathrm{R}}=83\right.$ ps.amagat $)$ measured at lower pressure in the same gas mixture by investigating the field-free alignment signal with a standard (two pulses) pump-probe technique [19]. The good match between the experimental values, that are both well reproduced by the CMDS model, reveals that the decay rates of the revival and echo are comparable for the $\mathrm{CO}_{2}-\mathrm{He}$ collisions. In order to figure out if this finding applies to other high-pressure gases, we performed measurements of rotational echoes in pure $\mathrm{CO}_{2}$ for which the decay rate of the alignment revivals have also been measured [19] and computed [18]. The corresponding decay rate of the echoes is presented in Fig. 3 (c) together with the CMDS simulations. The data summarized in Table I show that the dissipation of the echo and alignment revivals in pure $\mathrm{CO}_{2}$ occurs with a comparable time con- 
stant, which is consistent with the results obtained in the gas mixture. However, note that the CMDS reproduce more closely the experimental value in $\mathrm{CO}_{2}-\mathrm{He}$ than in pure $\mathrm{CO}_{2}$, which was already observed in probing the dissipation of molecular alignment revivals [18, 19]. It is worth noting that, for $\mathrm{CO}_{2}$, the decay rates of the echo, $\tau_{\mathrm{E}}$, and of the alignment revivals, $\tau_{\mathrm{R}}$, are close to that associated to the dissipation of the dipole autocorrelation function [26], $\tau_{\mathrm{D}}$ (as can be obtained through $\tau_{\mathrm{D}}=\left(2 \pi c<\gamma_{\mathrm{B}}>\right)^{-1}$, where $\gamma_{\mathrm{B}}$ is the average pressure broadening coefficient in $\mathrm{cm}^{-1} /$ amagat in the spectral domain). Indeed, experiments yield $\tau_{\mathrm{D}}=51.2$ ps.amagat for pure $\mathrm{CO}_{2}$ [27] and 82.3 ps.amagat for $\mathrm{CO}_{2}-\mathrm{He}[28,29]$, while direct calculations lead to very close values of 51.3 ps.amagat [26] and 79.6 ps.amagat [29], respectvely. The finding that $\tau_{\mathrm{E}} \approx \tau_{\mathrm{R}} \approx \tau_{\mathrm{D}}$ for $\mathrm{CO}_{2}$ and $\mathrm{CO}_{2}-\mathrm{He}$ is in apparent disagreement with the recently measured value $\tau_{\mathrm{E}}=13$ ps.agamat for pure OCS gas [20], about 2.5 times smaller than the experimental values $\tau_{\mathrm{R}}=32.4$ ps.amagat [30] and $\tau_{\mathrm{D}}=31.4$ ps.amagat $[31,32]$. We have no explanation for this difference that is not reproduced by direct calculations [22], which lead to $\tau_{\mathrm{E}}=45$ ps.amagat, $\tau_{\mathrm{R}}=36.0$ ps.amagat, and $\tau_{\mathrm{D}}=34$ ps.amagat, and deserves new experimental investigations. The dissipation of the echoes and revivals both result from collision-induced changes of the molecular rotational speeds. The associated decay time constants are expected to be of the same order, as confirmed by the present results. However, differences may arise, depending on the collisional pairs, due to the different nature (classical and quantum) of these two alignment signatures.

The present Letter shows that rotational alignment echoes represent so far a unique method for probing ultrafast rotational relaxation for elevated molecular densities in systems for which "usual" alignment revivals cannot be observed anymore. A first natural and tempting extension of the present study would thus be to carry similar investigations in the liquid phase for which any rotational information or coherence imprinted in the system has vanished due to very quick thermalization at time scales of a few ps. In principle, femtosecond time scales should be accessible by reducing the duration of the laser pulses. Similarly, it would be very interesting to apply rotational echoes to molecules embedded in helium nanodroplets. For instance, collective rotation of iodine molecule and its solvation shell can lead to fieldfree molecular alignment revivals [33]. Very recently, it has been demonstrated that 1D or 3D alignment of large and complex molecules inside helium nanodroplets could persist for several tens of ps after the rapid truncation of a strong laser pulse [34]. Echoes could therefore provide a mean for real-time studies of nonequilibrium solutesolvent ultrafast dynamics.

This work was supported by the Conseil Régional de Bourgogne (PARI program), the CNRS, the FEDERFSE Bourgogne 2014/2020, and the EIPHI Gradu- ate School (contract "ANR-17-EURE-0002"). We appreciate the support by the National Key R\&D Program of China (Grant No. 2018YFA0306303), the National Natural Science Foundation of China (Grant Nos. 11425416, 11834004 and 11761141004), the 111 Project of China (Grant No. B12024), the Israel Science Foundation (Grant No. 746/15), the ICORE program "Circle of Light", and the ISF-NSFC program (Grant No. 2520/17). J. Ma acknowledges the support from the China Scholarship Council (CSC). I.A. acknowledges support as the Patricia Elman Bildner Professorial Chair. This research was made possible in part by the historic generosity of the Harold Perlman Family.

* Electronic address: olivier.faucher@u-bourgogne.fr

$\dagger$ Electronic address: jwu@phy.ecnu.edu.cn

\# Electronic address: ilya.averbukh@weizmann.ac.il

[1] E. L. Hahn, Phys. Rev. 80, 580 (1950).

[2] E. L. Hahn, Phys. Today 6, 4 (1953).

[3] N. A. Kurnit, I. D. Abella, and S. R. Hartmann, Phys. Rev. Lett. 13, 567 (1964).

[4] I. D. Abella, N. A. Kurnit, and S. R. Hartmann, Phys. Rev. 141, 391 (1966).

[5] R. M. Hill and D. E. Kaplan, Phys. Rev. Lett. 14, 1062 (1965).

[6] R. W. Gould, T. M. O'Neil, and J. H. Malmberg, Phys. Rev. Lett. 19, 219 (1967).

[7] G. Stupakov, Echo Effect in Hadron Colliders (SSC Report SSCL-579, 1992).

[8] G. Stupakov and S. Kauffmann, Preprint SSCL-587, Superconducting Supercollider Laboratory (1992).

[9] J. H. Eberly, N. B. Narozhny, and J. J. SanchezMondragon, Phys. Rev. Lett. 44, 1323 (1980).

[10] J. Parker and C. R. Stroud, Phys. Rev. Lett. 56, 716 (1986).

[11] I. S. Averbukh and N. Perelman, Physics Letters A 139 , 449 (1989).

[12] R. Robinett, Physics Reports 392, 1 (2004).

[13] G. Karras, E. Hertz, F. Billard, B. Lavorel, J. M. Hartmann, O. Faucher, E. Gershnabel, Y. Prior, and I. S. Averbukh, Phys. Rev. Lett. 114, 153601 (2015).

[14] G. Karras, E. Hertz, F. Billard, B. Lavorel, G. Siour, J. M. Hartmann, O. Faucher, E. Gershnabel, Y. Prior, and I. S. Averbukh, Phys. Rev. A 94, 033404 (2016).

[15] K. Lin, P. Lu, J. Ma, X. Gong, Q. Song, Q. Ji, W. Zhang, H. Zeng, J. Wu, G. Karras, et al., Phys. Rev. X 6, 041056 (2016).

[16] K. Lin, J. Ma, X. Gong, Q. Song, Q. Ji, W. Zhang, H. Li, P. Lu, H. Li, H. Zeng, et al., Opt. Express 25, 24917 (2017).

[17] D. Rosenberg, R. Damari, S. Kallush, and S. Fleischer, J. Phys. Chem. Lett. 8, 5128 (2017).

[18] J.-M. Hartmann, C. Boulet, T. Vieillard, F. Chaussard, F. Billard, O. Faucher, and B. Lavorel, J. Chem. Phys. 139, 024306 (2013).

[19] T. Vieillard, F. Chaussard, F. Billard, D. Sugny, O. Faucher, S. Ivanov, J. M. Hartmann, C. Boulet, and B. Lavorel, Phys. Rev. A 87, 023409 (2013). 
[20] D. Rosenberg, R. Damari, and S. Fleischer, Phys. Rev. Lett. 121, 234101 (2018).

[21] H. Stapelfeldt and T. Seideman, Rev. Mod. Phys. 75, 543 (2003).

[22] See Supplemental Material at http://link.aps.org/ for details about the experimental setup, the interplay between echoes and imaginary echoes, and the CMDS model.

[23] D. P. Shelton, Phys. Rev. A 42, 2578 (1990).

[24] Y. Itikawa, J. Phys. Chem. Ref. Data 31, 749 (2002).

[25] S. Ramakrishna and T. Seideman, Phys. Rev. Lett. 95, 113001 (2005).

[26] J. M. Hartmann, H. Tran, N. H. Ngo, X. Landsheere, P. Chelin, Y. Lu, A. W. Liu, S. M. Hu, L. Gianfrani, G. Casa, et al., Phys. Rev. A 87, 013403 (2013).

[27] A. Predoi-Cross, A. V. Unni, W. Liu, I. Schofield, C. Holladay, A. R. W. McKellar, and D. Hurtmans, J. Mol. Spectrosc. 245, 34 (2007).

[28] F. Thibault, J. Boissoles, R. Le Doucen, J. P. Bouanich,
P. Arcas, and C. Boulet, J. Chem. Phys. 96, 4945 (1992).

[29] F. Thibault, B. Calil, J. Boissoles, and J. M. Launay, Phys. Chem. Chem. Phys. 2, 5404 (2000).

[30] R. Damari, D. Rosenberg, and S. Fleischer, Phys. Rev. Lett. 119, 033002 (2017).

[31] S. Matton, F. Rohart, R. Bocquet, G. Mouret, D. Bigourd, A. Cuisset, and F. Hindle, J. Mol. Spectrosc. 239, 182 (2006).

[32] M. A. Koshelev, M. Y. Tretyakov, F. Rohart, and J.-P. Bouanich, J. Chem. Phys. 136, 124316 (2012).

[33] B. Shepperson, A. A. Sondergaard, L. Christiansen, J. Kaczmarczyk, R. E. Zillich, M. Lemeshko, and H. Stapelfeldt, Phys. Rev. Lett. 118, 203203 (2017).

[34] A. S. Chatterley, C. Schouder, L. Christiansen, B. Shepperson, M. H. Rasmussen, and H. Stapelfeldt, Nat. Commun. 10, 133 (2019). 